\section{Familienergänzende Kinderbetreuung: Schaden oder Chance für das Kind?}

\author{
A. Karsh
}

In den Vereinigten Staaten sind heutzutage $7 \frac{1}{2}$ Millionen Kleinkinder in nichtmütterlicher Betreuung. Über 60\% der Mütter mit Kindern unter 6 Jahren arbeiten zumindest teilzeitig ausser Hause. Für viele Leute ist diese Zahl eine Alarmglocke, und Fachleute wie Laien fragen sich, ob diese Entwicklung der jüngsten Generation Schaden bringen wird. Auch in der Schweiz gibt es immer mehr Mütter von Kleinkindern, die ausser Hause arbeiten. Es gibt verschiedene Gründe, warum Mütter heutzutage voll- oder teilzeitig arbeiten. Finanzielle Gründe sind unter anderem, dass der Lohn des Vaters alleine nicht ausreicht, um die Familie über der Armutsgrenze zu halten, dass die Mutter alleinerziehend ist, oder dass die Eltern ihren Lebensstil nicht ändern möchten mit der Geburt eines Kindes. Ideelle Gründe sind etwa die Karriere der Frau, die sie nicht aufgeben möchte, oder die Überzeugung einer Mutter, dass sie eine bessere Mutter sei, wenn sie nicht 24 Stunden mit dem Kinde zu Hause ist und womöglich depressiv wird. Was immer die Gründe sein mögen, einige Fachleute fingen vor etwa 10 Jahren an zu vermuten, dass familienergänzende Kinderbetreuung schädlich sein könnte für die Kinder, und jene Kontroverse unter Experten war der Grund, warum die «NICHD Study of Early Child Care» ins Leben gerufen wurde.

\section{NICHD}

NICHD steht für «National Institute of Child Health and Human Development", ein Departement des Gesundheitsministeriums der USA. Entwicklungspsychologen an zehn Universitäten verfolgen seit 1991 die Entwicklung von ca. 1400 Kindern in zehn städtischen und ländlichen Gemeinden. Die Demographie der Familien reflektiert ziemlich gut die Zusammensetzung der Bevölkerung der USA. Ausgeschlossen bei der Rekrutierung wurden "High-risk»-Familien

Korrespondenz:

Andrea Karsh, lic. phil.

University of California

NICHD Study of Early Child Care

3340 Social Ecology II

USA-Irvine, CA 92697-7085 sowie Familien, die wir aus praktischen Gründen wenig gut verfolgen könnten. Ansonsten ist die Studie repräsentativ. Ein weiteres Charakteristikum der Untersuchung ist, dass sie breit angelegt ist, was heisst, dass alle möglichen nichtmütterlichen Betreuungspersonen befragt und beobachtet wurden: Väter, Grosseltern, andere Verwandte, Au-pair-Mädchen, Babysitter, Tagesmütter und Krippenpersonal. Solange das Kind mindestens zehn Stunden pro Woche in einem Arrangement weilte, untersuchten wir den Betreuungsplatz. Weiter handelt es sich bei der "NICHD Study of Early Child Care" um eine Langzeitstudie. Wir verfolgen die Familien von der Geburt des Kindes bis zu dessen Pubertät; vielleicht sogar weiter. Im Vergleich $\mathrm{zu}$ anderen Untersuchungen ist diese prospektiv, d.h. wir wussten nicht zum voraus, welche Betreuungsarten die Familie wählen würde. Schliesslich ist die Studie umfassend, d.h. wir sind mit den Familien alle drei Monate in Kontakt, und die Qualität der Betreuung wird ausgiebig analysiert: Während Ausmass (Anzahl Stunden pro Woche), Stabilität (Häufigkeit des Wechsels) des Betreuungsplatzes, sowie Art der Betreuung von der Mutter angegeben und von den Betreuungspersonen bestätigt wurden, evaluierten wir die Qualität der Betreuung folgendermassen: Verhältnis Erwachsene zu Kindern, Gruppengrösse, Ausbildung der Betreuungspersonen, Erfahrung der Betreuungspersonen, Einstellung der Betreuer, wie Kinder erzogen werden sollen, Einstellung der Betreuer zu ihrem Beruf (professionell?), physisches Umfeld (sauber, sicher, kinderfreundlich, Materialienvielfalt, etc.), und schliesslich die Beobachtung vor Ort durch qualifizierte Forscher. Die stundenlangen Beobachtungen beinhalteten Verhaltensfrequenzen, indem positive und negative Verhaltensweisen der Betreuungspersonen addiert wurden, sowie qualitative Skalen, welche Verhalten und Eigenschaften der Kinder und Betreuungspersonen aufzeigten.

\section{Soziale und emotionale Entwicklung}

Verschiedene Studien haben gezeigt, dass die Qualität der Beziehung des Kleinkindes zu seiner Mutter ein ausgezeichneter Prädiktor für seine emotionale und soziale Entwicklung ist. Kinder mit sicherem Bindungsverhalten sind später sozial versierter, haben ein besseres Selbstvertrauen und bessere Beziehungen zu Kameraden. Gemessen wird das Bindungsverhalten in einem standardisierten und anerkannten Verfahren, genannt "strange situation", in dem das Kind kurz von der Mutter getrennt wird, um dann das Verhalten des Kindes bei der Wiederkehr der Mutter zu evaluieren. Grüsst das Kind die Mutter freudig, umarmt sie gar, so zeigt dies ein sicheres Bindungsverhalten. Wendet sich das Kind von der Mutter ab, oder ist es gar wütend auf sie, so ist sein Bindungsverhalten unsicher. Ungefähr zwei Drittel aller USKinder zeigen ein sicheres Bindungsverhalten.

Die Auswertung unserer Daten zeigt keine statistisch signifikanten Auswirkungen von Fremdbe- 
treuung auf die Mutter-Kind-Beziehung. Weder die Qualität der Betreuung, die Anzahl Stunden, das Eintrittsalter, die Wechselhäufigkeit, noch die Art der Betreuung zeigen Unterschiede zwischen Kindern in Fremdbetreuung und Kindern, die ausschliesslich von der Mutter betreut werden. - Es gibt aber folgende statistisch signifikanten Beziehungen: Wenn die Mutter wenig sensitiv ist, d.h. wenn die Betreuung $z u$ Hause qualitativ schlecht ist, und das Kind zugleich in qualitativ schlechter Fremdbetreuung ist, dann ist es wahrscheinlicher, dass die Mutter-Kind-Beziehung negativ ist (bloss 45\% sicheres Bindungsverhalten). Und wenn die Mutterbetreuung schlecht ist, und das Kind zugleich mehr als zehn Stunden in Fremdbetreuung verbringt, dann ist ein sicheres Bindungsverhalten ebenfalls gefährdet. Auch ein häufiger Wechsel der Betreuungsplätze in Verbindung mit wenig sensitiver Mutterbetreuung vermindert die Wahrscheinlichkeit eines sicheren Bindungsverhaltens. Kurz, die Art und Weise, wie die Mutter mit dem Kinde umgeht, wirkt sich primär auf die Qualität der Mutter-Kind-Beziehung aus. Schlechte Mutterbetreuung in Verbindung mit wenig idealer Fremdbetreuung kann sich negativ auf die Kinder auswirken.

Wir fanden weiterhin keine Unterschiede zwischen fremdbetreuten und mutterbetreuten Kindern in emotionaler Anpassung, Gehorsamkeit, Verhaltensproblemen, noch in sozialem Verhalten mit anderen Kindern. Hingegen ist die Qualität der Betreuung - ob zu Hause oder in Fremdbetreuung - prognostisch für das Verhalten des Kindes. Verhaltensprobleme zeigen sich häufiger bei Kindern in qualitativ minderwertigeren Arrangements. Die Interviews mit Betreuern ergaben auch, dass Kinder im Vorschulalter weniger Verhaltensprobleme haben und kooperativer sind, wenn sie ihren Tag in Gruppen von mindestens vier Kindern verbrachten, also in Krippen, Horten oder grösseren Tagesmutterarrangements. Anscheinend lernen Kinder in Gruppen besser - oder früher - wie man mit anderen umgeht, bzw. umgehen soll.

\section{Kognitive und Sprachentwicklung}

\section{Gehen Familieneinflüsse verloren?}

Ein Bedenken vieler Eltern ist, dass ihr Kind in Fremdbetreuung weniger kognitive Stimulation erhält als es zu Hause von den Eltern erhalten würde. Um herauszufinden, ob die kognitive und Sprachentwicklung der Kinder verlangsamt oder allenfalls behindert wird durch Fremdbetreuung, wählten wir diejenigen Kinder in unserer Studie aus, die ausschliesslich von der Mutter betreut wurden, d.h. nie mehr als zehn Stunden pro Woche in nichtmütterlicher Betreuung verbrachten, und verglichen diese Kinder mit denjenigen, die vom 4. Lebensmonat an mindestens 30 Stunden pro Woche in Fremdbetreuung verbrachten. Verglichen wurde die Sprach- und kognitive Entwicklung im 24. und 36. Altersmonat. - Wir fanden keinen statistisch signifikanten Unterschied in der Sprach- und kognitiven Entwicklung der Kinder in den beiden Gruppen. Dies bedeutet, dass der Einfluss der Familie nicht verloren geht, wenn die Kinder den Tag ausser Hause, bzw. in nichtmütterlicher Betreuung verbringen. Dieses Resultat geht Hand in Hand mit unserer Schlussfolgerung, dass Familienvariablen statistisch gesehen mindestens doppelt so stark verantwortlich sind für Unterschiede in der kindlichen Entwicklung als Betreuungscharakteristika.

Obwohl verallgemeinernd keine Unterschiede gefunden wurden, fanden wir die folgenden statistisch signifikanten Tendenzen: Kinder in einem Haushalt mit bloss einem Elternteil, die ausschliesslich von der Mutter betreut werden, haben schlechtere Schuleintrittstestresultate. Kinder, deren Mütter psychische Probleme haben (Depression, negative Einstellungen, etc.), und die ausschliesslich von der Mutter betreut werden, zeigen ein niedereres Niveau in ihrer Sprachund kognitiven Entwicklung. Weiter fanden wir, dass Frauen, die glauben, dass es gut sei, wenn eine Mutter arbeiten geht, Kinder mit besseren Schuleintrittstestresultaten hatten, sofern die Kinder vollzeitig fremdbetreut wurden. Auch Mütter, die modernere Erziehungseinstellungen haben (z.B. weniger autoritärer Stil), und deren Kinder vollzeitig fremdbetreut werden, waren in ihrer Sprach- und kognitiven Entwicklung weiter.

\section{Fremdbetreuung versus Mutterbetreuung}

Kinder, die ausschliesslich von der Mutter betreut werden, sind verallgemeinernd weder besser noch schlechter in der Sprach- und kognitiven Entwicklung als Kinder, die teil- oder vollzeitig von anderen Personen betreut werden. (Statistische Bemerkung: Um nachzuweisen, was für Auswirkungen familienergänzende Kinderbetreuung per se hat, mussten Familienprädiktoren statistisch ausgeklammert [kontrolliert] werden.)

Wenn wir aber die Qualität der Betreuung betrachten, zeigen sich folgende Unterschiede: Kinder in qualitativ guter Fremdbetreuung schnitten besser ab als Kinder, die ausschliesslich von der Mutter betreut wurden. Und Kinder in qualitativ minderwertiger Fremdbetreuung schnitten schlechter ab als Kinder, die ausschliesslich von der Mutter betreut wurden. Gute Qualität am Betreuungsplatz ist also eine Chance, schlechte Qualität ein Risiko für das Kind. Schliesslich ist aber auch die Qualität der Mutterbetreuung ausschlaggebend für die kindliche Entwicklung: Kinder in qualitativ schlechter Mutterbetreuung hatten schlechtere Resultate als Kinder in schlechter Fremdbetreuung! Diese Resultate zeigen deutlich, dass wir nicht nur von der Qualität der Fremdbetreuung reden dürfen, sondern auch von der Qualität der Betreuung zu Hause.

\section{Quantität der Fremdbetreuung}

Unsere Resultate zeigen keine Unterschiede in der kognitiven und Sprachentwicklung zwischen Kindern, die viele Stunden fremdbetreut werden und Kindern, die wenige oder keine Stunden ausser Hause verbringen.

Die Sprach- und kognitive Entwicklung unserer Vorschulkinder wird durch die Qualität der Betreu- 
ung - ob mütterliche oder nichtmütterliche - beeinflusst. Kinder in guter Betreuung haben einen Vorsprung und somit einen Vorteil. Unter fremdbetreuten Kindern scheint für die Sprach- und kognitive Entwicklung der Betreuungsort Krippe/Kinderhort ein Vorteil zu sein gegenüber Betreuung durch Babysitter, Haushaltshilfe, Tagesmutter, Grosseltern oder andere Betreuung zu Hause. Wir erklären die Entwicklungsvorsprünge der Krippenkinder dadurch, dass Kinder in Krippen und Horten vermehrt kognitiver Stimulation und lernorientierten Materialien ausgesetzt sind als Kinder zu Hause.

\section{Physische Gesundheitsentwicklung}

Nun zu den eben erschienenen Resultaten über mögliche Auswirkungen familienergänzender Betreuung auf die physische Gesundheitsentwicklung des Kindes. Über die Jahre hinweg fragten wir die Mütter alle drei bis sechs Monate nach dem Gesundheitszustand der Kinder. Wir erfassten Unfälle, fragten nach Otitiden, Magen-/Darmproblemen, Oberen Luftwegsinfekten, und notierten akute und chronische Krankheiten jeder Art. Von Zeit zu Zeit überprüften wir die Aussagen der Mütter mit den Kinderärzten, wobei wir eine erstaunlich hohe Übereinstimmung fanden. Es war daher möglich, die Aussagen der Mütter als valide anzunehmen und auf ausführliche Ärztekrankengeschichten zu verzichten.

Das Risiko, an Otitiden zu erkranken, ist mehr als doppelt so hoch für einjährige Kinder, die in einer Krippe betreut werden, als für Kinder, die zu Hause betreut werden. Wenn sie aber drei Jahre alt sind, ist diese Wahrscheinlichkeit nur noch 28\% höher. Auch die Wahrscheinlichkeit für Obere Luftwegsinfekte ist höher für Krippenkinder als für zu Hause betreute Kinder. Ein- und zweijährige Kinder in Fremdbetreuung ausser Hause sind deutlich anfälliger; diese Unterschiede verschwinden aber im dritten Lebensjahr. Daraus lässt sich schliessen, dass Kinder in Betreuung ausser Hause mit der Zeit resistenter werden gegenüber Ansteckungskrankheiten. Es wird interessant sein herauszufinden, ob diese Kinder in Schulalter weniger häufig krank sind als ihre Kameraden, die ausschliesslich zu Hause waren bis zum Kindergartenalter.

Je grösser die Anzahl Kinder am Betreuungsort, und je grösser die Fremdbetreuung in Stunden pro Woche, desto häufiger sind die Kinder krank, besonders in den ersten zwei Lebensjahren. Kleinkinder in Krippen leiden am häufigsten an Otitiden und Luftwegsinfekten. Die Tatsache, dass Krippenkinder am häufigsten krank sind, lässt vermuten, dass das Ansteckungsrisiko primär ausschlaggebend ist für diese Krankheiten.

Wir fragten uns weiter, ob die Krankheiten in erster Linie auf die erhöhte Ansteckungsgefahr im Umgang mit anderen Kindern zurückzuführen seien, oder ob auch andere Faktoren wie Frühgeburt, Stillen versus Flaschennahrung, Familienzusammensetzung oder stressvolle Lebensveränderungen eine
Rolle spielen? Familienvariablen wie Armut, Ausbildung der Mutter, Familiengrösse, Alleinerziehende oder beide Eltern im Haushalt standen in keiner Beziehung zur Krankheitshäufigkeit. Frühgeburten sowie Kinder, welche nicht gestillt wurden, hatten kein erhöhtes Krankheitsrisiko (notabene: Kinder mit Geburtskomplikationen wurden im Sample ausgeschlossen!). Wir fanden auch keinen Hinweis darauf, dass Stress im Familienleben eine signifikante Rolle spielt für die Gesundheit der Kinder. Situationen wie Partnerwechsel, Geburt eines Geschwisters, Umzug, Wechsel in der Betreuungssituation und ähnliches scheinen keinen direkten Einfluss zu haben auf die physische Gesundheit der Kinder.

Unsere Datenanalyse ergab weiter, dass die häufigeren Krankheitsfälle von fremdbetreuten Kindern keinen schädlichen Einfluss auf deren Sprachentwicklung, Schuleintrittsreife und soziales Verhalten haben. Kinder mit häufigeren Krankheiten zeigen keine Entwicklungsrückstände oder Verhaltensprobleme. Auch Kinder mit Trommelfellröhrchen entwickeln sich nicht anders als ihre Kameraden.

Zusammenfassend: Unsere Studie zeigt, dass das Ansteckungsrisiko primär verantwortlich ist für die häufigeren Krankheiten fremdbetreuter Kinder in den ersten Lebensjahren. Kinder in familienexterner Betreuung sind in den ersten zwei Lebensjahren häufiger krank, was aber keine negativen Konsequenzen hat für ihre normale Entwicklung. Es wird interessant sein zu erfahren, ob fremdbetreute Kinder später, im Schulalter, weniger anfällig sind für ansteckende Krankheiten.

\section{Zusammenfassung}

Die Resultate der "NICHD Study of Early Child Care» zeigen, dass Fremdbetreuung per se dem Kinde nicht schadet. Qualitativ gute Betreuung hat positive Auswirkungen auf die kognitive und Sprachentwicklung, wobei zu betonen ist, dass Betreuungsfaktoren sekundär verantwortlich sind für die kindliche Entwicklung, während primär Familienvariablen wie Ausbildung, Intelligenz, Einstellungen und Sensitivität der Mutter/Eltern sowie das Familieneinkommen ausschlaggebend sind. Qualitativ schlechte Fremdbetreuung oder mehr als zehn Stunden Fremdbetreuung pro Woche in Verbindung mit einer wenig sensitiven Mutter erhöhen das Risiko, dass das Kind Probleme hat in seiner Entwicklung. Für die Sprachund kognitive Entwicklung scheinen Krippenkinder einen Vorteil zu haben (Krippen und Horte lehren Buchstaben und Zahlen), und für die soziale Entwicklung haben offenbar Kinder einen Vorteil, die ihren Tag mit mehreren anderen Kindern verbringen. Ein- und zweijährige Kinder in Betreuung ausser Hause sind eher krank; ihre Entwicklung scheint aber dadurch nicht schädlich beeinflusst zu sein.

All dies zeigt deutlich, dass Eltern keine Schuldgefühle haben sollen, wenn beide Eltern ausser Hause arbeiten, besonders dann nicht, wenn die Kinder in qualitativ guter Betreuung sind. Unsere Resultate sind 
aber auch "good news» für all die Mütter, die bewusst zu Hause bei ihren Kindern bleiben und ihren Beruf aufgeben, denn qualitativ gute Mutterbetreuung - ob voll- oder teilzeitig - ist der wichtigste Faktor für eine gesunde Entwicklung des Kindes.

\section{Was bedeuten die Resultate für den Kinderarzt im nächsten Jahrtausend?}

Angenommen, jeder Pädiater hat das ganzheitliche Wohl des Kindes als Ziel, und nicht nur die medizinische Gesundheit, so denke ich, der Pädiater muss den Fokus wechseln, weg vom Kinde, auf die ganze Familie. Für viele mag es gesunder Menschenverstand sein, dass Familienvariablen primär für die gesunde Entwicklung des Kindes verantwortlich sind. Ich stimme damit überein. Aber jetzt haben wir auch die Beweise, die statistischen Fakten dazu. Müsste ich die Resultate in einem Satz zusammenfassen, dann wäre das "Es kommt auf die Famile an" oder "family matters". Was das Kind braucht, sind psychisch gesunde, glückliche, und feinfühlige Eltern. Dies bedeutet für den Kinderarzt, der ja oft die einzige Fachperson ist, die die jungen Eltern in den ersten Lebensjahren ihres Kindes relativ regelmässig sehen, dass nach dem Wohlergehen der Eltern gefragt werden soll, dass Elternberatungsstellen angepriesen werden, dass den Eltern klar gemacht wird, dass es im besten Interesse des Kindes ist, wenn sie sich bei Problemen an eine Fachstelle wenden, dass der Mutter gesagt wird, dass es OK ist, wenn sie arbeiten gehen möchte, usw. Weiter denke ich, dass Pädiater enger zusammenarbeiten sollten mit Beratungsstellen und Elternweiterbildungsinstituten. Dies ist sicher nicht einfach, wenn man bedenkt, wie häufig sich gerade jene Stellen den Ärzten und anderen Akademikern gegenüber eher zurückhaltend zeigen ... Abschliessend denke ich, dass der Pädiater auch auf gesellschaftlich-politischer Ebene einen Einfluss haben kann im Interesse des Kindes. Vertreten Sie öffentlich die Resultate unserer Studie, setzen Sie sich ein für qualitativ bessere Betreuung, für Qualitätskontrollen aller Arten von
Betreuung, nicht nur von öffentlichen Tageskrippen. Stimmen Sie für Elternschulung für alle jungen Paare, für Kurse, die Eltern lehren, die Qualität zu Hause zu verbessern. Helfen Sie mit, die Armut zu bekämpfen und die Ausbildung für alle zu verbessern. Helfen Sie mit, dem Kinde das zu geben, was es für einen geglückten Start ins Leben am meisten braucht: ein gesundes Elternhaus, eine glückliche Mutter, und qualitativ gute Betreuung.

\section{Literatur}

1 NICHD Early Child Care Research Network. Characteristics of Infant Child Care: Factors Contributing to Positive Caregiving. Early Childhood Research Quarterly 1996;11(3):269-306.

2 NICHD Early Child Care Research Network. Familial Factors Associated with Characteristics of Nonmaternal Care for Infants. Journal of Marriage and Family 1997;59(2):389-408.

3 NICHD Early Child Care Research Network. Child Care and Child Development. The NICHD Study of Early Child Care. In: Friedman SL, Haywood HC (eds.). Developmental FollowUp: Concepts, Domains and Methods New York: Academic Press; 1994. p. 377-96.

4 NICHD Early Child Care Research Network. Child Care and Common Communicable Illnesses: Results from the NICHD Study of Early Child Care. 1999 (submitted for publication).

5 NICHD Early Child Care Research Network. The Effects of Infant Child Care on Attachment Security: Results of the NICHD Study of Early Child Care. Child Development 1997; 68(5):860-79.

6 NICHD Early Child Care Research Network. Child-Outcomes When Child Center Classes Meet Recommended Guidelines for Quality. Paper presented at the Annual Meeting of State Child Care Administrators, Child Care Bureau, Administration for Children and Families, USDHHS, Washington, DC. 1999.

7 NICHD Early Child Care Research Network. Child-Care and Mother-child Interaction in the first three years of life. Developmental Psychology 1999;35(6):1399-413.

8 NICHD Early Child Care Research Network. Early Child Care and Self-Control, Compliance and Problem Behavior at twenty-four and thirty-six months. Child Development 1998;69(3):1145-70.

9 NICHD Early Child Care Research Network. The Relation of Child Care to Cognitive and Language Development. Child Development 1999.

10 NICHD Study of Early Child Care and Youth Development webpage: http://public.rti.org/secc 